\title{
Transition of primary to secondary cell wall synthesis
}

Zheng Li $^{1}$, Alisdair R. Fernie ${ }^{2}$, Staffan Persson ${ }^{1,3 \#}$

${ }^{1}$ School of Biosciences, University of Melbourne, Parkville VIC 3010, Melbourne, Australia

${ }^{2}$ Max-Planck-Institute of Molecular Plant Physiology, Am Muehlenberg 1, 14476 Potsdam-

Golm, Germany

${ }^{3}$ ARC Centre of Excellence in Plant Cell Walls, School of Biosciences, University of

Melbourne, Parkville VIC 3010, Melbourne, Australia

\#Corresponding author:

Staffan Persson

School of Biosciences

University of Melbourne,

VIC 3010, Melbourne,

Australia

Email: Staffan.persson@unimelb.edu.au 


\begin{abstract}
The construction of a secondary cell wall is an important and necessary developmental decision that supports cell function and plant stature. Unlike the primary cell walls, which are initiated during cell division and develop along with the expansion of the cells, secondary cell walls are constructed after the cells have stopped growing. Hence, the transition from primary to secondary wall synthesis marks an important and distinct metabolic investment by the plant. This transition requires a coordinated change of a plethora of cellular processes, including hormonal, transcriptional and post-transcriptional activities, metabolic flux redistributions and enzymatic activities. In this review, we briefly summarize the hormonal and transcriptional control of the primary to secondary wall transition, and highlight important gaps in our understanding of the metabolic framework that support the transition. Several tools that may aid in future research efforts to better understand the changes in cell wall synthesis during the trans-differentiation are also discussed.
\end{abstract}

\title{
1 Introduction
}

A major developmental difference between animals and plants is that plant cell morphogenesis and pattern formation are accomplished largely through asymmetric cell divisions and cell enlargement $[1,2]$. These events are underpinned by extensive synthesis and re-modelling of the plant cell wall, a glycan-enriched extracellular structure. In the course of cell division, a nascent cell wall is deposited usually perpendicular to the dividing axis of a mother cell following mitosis $[1,3]$. The completion of this cross wall marks the end of cytokinesis and thus the formation of two daughter cells. Importantly, the position of the cross wall may be regarded as a first "decision point" of cell fate as it results in two daughter cells with different shape [1]. After cytokinesis, the two daughter cells typically expand to obtain their final form and function. Cell expansion requires rapid synthesis, localized deposition, and extensive re-modelling of cell wall material to allow anisotropic cell growth. One impressive example of cell form and function is the dramatic differentiation of the xylem tissue [4]. Xylem vessels consist of interconnected cells that can reach over a meter in length, which is approximately 10,000 times larger than the size of newborn cells [5]. However, the elongation of these cells, and their interconnectedness, are not sufficient to ensure solute transport through the vessels. To sustain this capacity, the maturing vessel cells produce a secondary and much thicker cell wall. Due to the developmental order, these two types of cell walls were historically termed as primary and secondary cell walls (PCWs and SCWs), respectively.

SCWs are specialized cell wall structures that support plant stature and cell functions [6, 7]. While SCWs are deposited around xylem vessels, they may also be found at anther endothecium, and around tracheids and fibres in woody tissues. The SCW structure represents a key advance for plants to adapt to terrestrial environments, and was a necessity for land plants to evolve. The PCWs of most plants consist of three major glycan structures; cellulose, hemicelluloses and pectins. The relative amounts of these three components can vary depending on species, on cell and tissue types, and on the developmental and environmental contexts [8]. For example, a typical dicot primary wall consists of cellulose, 
xyloglucans, a mixture of homo- and rhamnogalacturonans, and heavily glycosylated protein [8]. While the SCWs also contain large amounts of cellulose, they have a different set of hemicelluloses and reduced amounts of pectins [7]. Hence, the SCWs typically contain xylans and mannans, but also a significant amount of the polyphenolic structure lignin [7]. In contrast to PCWs, which are relatively elastic due to effective cell wall re-modelling, SCWs are characterised by their high rigidity. In both cell wall types, cellulose provides the main mechanical strength and steers plant cell morphology. For example, in the SCW, cellulose prevents vessels from collapsing due to the negative pressure inside of the cells [7]. Nevertheless, both xylan and lignin provide SCW strength as they aid in cross-linking cellulose microfibrils, and to an interconnected SCW framework. In addition, lignin confers water-repellent characteristics to the SCWs due to its inherent hydrophobicity and is thus crucial for the water transporting efficiency of the vessels [9]. Understanding how plants produce SCWs is therefore of significant biological interest.

Apart from the biological significance, SCWs also constitute the bulk of biomass a plant produces. This biomass is of major economic importance as it is used for a variety of industries, such as textile, lumber, feed and fuel $[8,10,11]$. While we understand a great deal of the enzymes involved in SCW synthesis, and how the corresponding genes are transcriptionally regulated, we know surprisingly little concerning the transition of the primary to SCW synthesis. That is, what aspects of the PCW synthesis are shut down when SCW synthesis is turned on, and what are the drivers for these processes? How is the metabolic framework changing during this transition? And, how can we begin to address these questions with the tools we currently have at hand? Here, we highlight aspects that are related to some of these questions, and also attempt to underline tools that may be used for future endeavours in this direction. With a few exceptions, this review mainly focuses on tracheary elements and xylem/interfascicular fibres due to length restrictions.

\section{Hormonal control of the initial onset of secondary wall production}

Almost every aspect of plant growth and development is under the control of various hormones [12-15]. It is therefore not surprising that also the transition from the primary to the SCW is controlled by their coordinated action $[16,17]$. Given the pleiotropic effect of single hormones, and the added combinatorial effects of multiple ones, it is difficult to dissect aspects of SCW formation the hormones regulate. However, it is clear that auxin, cytokinin and brassinosteroids contribute to the onset of SCW in vessels of Arabidopsis thaliana [1820]. Various combinations of these hormones have also been used to induce SCW synthesis in cell suspension cultures, e.g. in Zinnia elegans and Arabidopsis [21-24], supporting an active role of them in SCW production.

Several vessel-specific transcription factors (TFs) that drive SCW synthesis are under the control of auxin, cytokinin and brassinosteroids. These include the central NAC TFs, VASCULAR-RELATED NAC-DOMAIN (VND)6 and VND7 that are referred to as master TFs as expression of them in cells that do not normally undergo SCW synthesis triggers SCW production [7, 25]. Notably, robust activation of these TFs requires the presence of auxin, 
cytokinins and brassinosteroids, while each hormone alone has no major impact [25] (Fig. 1). Other VND TFs, namely VND1-5 and the NAC SECONDARY WALL THICKENING PROMOTING FACTOR (NST)3/ SECONDARY WALL-ASSOCIATED NAC DOMAIN PROTEIN (SND)1, may be induced by abscisic acid (ABA) [26]. While the ABA induction of SCW inducing TFs is interesting it is currently not clear whether this activation is part of an in planta activation process [26] (Fig. 1). Based on recent reports it appears that there may be at least an additional layer between the hormone tier and the master TFs. For example, the expression of VND6 and VND7 is regulated via a feedback-loop by ASYMMETRIC LEAVESLIKE (ASL) 19 that in turn are controlled by the auxin induced AUXIN RESPONSE FACTOR7 (ARF7) [27] (Fig. 1).

Both primary and secondary wall cellulose synthesis is regulated by cortical microtubules that guide the cellulose synthase complex (CSC) [10, 28-31]. This guidance is essential to maintain cell wall strength, cell morphology and function [32-35]. The microtubule array undergoes a dramatic re-organization during the transition between the two wall types. In elongating cells, the microtubule array is evenly dispersed across the cell cortex and is typically transversely oriented with regards to the cell's growth axis [36]. However, during SCW synthesis the microtubules form distinct and evenly distributed bands and/or lattices that mark synthesis of cellulose and other cell wall components [30, 37-39] (Fig 1). Hormones, such as AUX, BR and GA, impact on the microtubule organization [40-42], which could influence the patterning of SCWs and might affect the transition from primary to secondary wall synthesis (Fig. 1). For example, auxin can either induce reorientation or depolymerize the microtubule cytoskeleton, possibly due to different experimental conditions and materials [43-47]. However, the mechanistic details behind the impact of hormones on microtubule organization and dynamics remain largely obscure. Perhaps the recently reported AUXIN BINDING PROTEIN (ABP)1-mediated effects on microtubules might shed light on the relationship between auxin and microtubule organization [48]. However, the importance of $\mathrm{ABP} 1$ in auxin signalling has been questioned [49, 50], so care has to be taken when evaluating this protein in context of microtubule organization [51]. Moreover, insights gained from salt stress-related changes in the SCW synthesis regulatory network, and COMPANION OF CELLULOSE SYNTHASE (CC) proteins that affect microtubule dynamics may provide some links between $\mathrm{ABA}$ and microtubule organization [52, 53].

\section{The secondary wall transcription factor network}

During the past two decades, the transcriptional framework that underpins SCW formation has been extensively investigated [54-57]. There are many excellent reviews that give detailed information about the action and regulation of the TFs involved in SCW synthesis, including [54-58]. We are therefore only briefly summarizing these aspects here (Fig. 1). The first identified "master" TFs were the VND1-7 [25, 59-61], and ectopic expression of VND6 and VND7 resulted in trans-differentiation of cells that normally do not undergo xylogenesis, including mesophyll cells. These studies concluded that VND6 and VND7 regulated metaand protoxylem formation, respectively, in both Arabidopsis and poplar [25]. Analogously, over-expression of the five other VND genes led to ectopic SCW deposition and activated the expression of secondary wall biosynthetic genes for cellulose, xylan and lignin [61]. The fact 
that these $V N D$ genes are preferentially expressed in developing vascular tissues suggested that other SCW-containing cell types need different TFs to turn on SCW biosynthesis. Indeed, in Arabidopsis, the two TFs NST1 and NST2 function redundantly to initiate SCW biosynthesis in anther endothecium [62], and SND1/NST1 controls SCW deposition in fibre cells [63]. The complex relationship amongst the "master" TFs is also reflected by the unexpected result that over-expression of SND1 or NST1 induced SCWs with a xylem-vessellike pattern (helical and reticulated) despite the fact that fibre SCWs are uniformly thickened [62-64]. Furthermore, the proposed roles of the "master switches" do not necessarily mean that they are the only responsible regulators that determine the SCW cell fate. This is illustrated by the fact that dominant repression of the TFs only resulted in a reduced SCW thickening, but the cell identity was not affected [25]. For example, xylem vessels still underwent elongation and programmed cell death in VND6 or VND7 dominant repression Arabidopsis plants [25]. However, it remains unclear if there are other regulators that impact on cell differentiation without turning on SCW biosynthesis. Also, whether the initiation of SCW formation is the signal for cell growth termination (i.e., the end of cell enlargement and PCW formation) or vice versa is still an open question.

The master TFs regulate an intricate network of downstream TFs and genes encoding biosynthetic and hydrolytic enzymes (Fig. 1). In this network, MYB TFs account for a majority of the targets. These TFs are mainly activated, directly or indirectly, by the master TFs [54] and positively regulate the expression of various cell wall related genes. Two of the most prominent MYBs in this network, MYB46 and MYB83, work downstream of the master TFs, but they are also capable of triggering the expression of the majority of the SCW biosynthetic genes. Thus, these MYBs are considered as the converging point of the SCW transcriptional network. However, this model has been challenged as there exist complex feedback (e.g., downstream TFs MYB4, MYB7, and MYB32 can repress SND1 independently of MYB46 and MYB83) and feed-forward loops in the SCW TF network [53, 65], and it is, therefore, possible that the inferred conversion point is overestimated (Fig. 1). These multiple layers of regulation might precisely determine structural features of the SCW, and perhaps also be beneficial for plants to endure abiotic stress [53]. It is interesting to note that some of the SCW related TFs may maintain or promote the expression of the "master" TFs via a positive feedback loop. For example, over-expression of the TF ASL19 led to activation of VND7 while it also is a direct target of VND7 [27]. Hence, ASL19 expression is therefore also induced by VND7. In addition to activation, transcriptional repression in the SCW regulatory framework has also been recently reported. VNI2 and WRKY12 are transcriptional repressors of $V N D 7$ and $N S T 2$, respectively [66, 67]. In addition, the recently identified E2Fc, an upstream regulator of VND6 and VND7, can act either as an activator or a repressor in a dose-dependent manner [53]. These types of relationships therefore add additional dimensions of control to the regulatory network, and support the notion that plants need a precise transcriptional regulatory system to control SCW formation.

\section{Metabolic transitions}

The formation of SCW is a very rapid and energy consuming process, so it is clear that a dramatic metabolic switch needs to occur to effectively supply precursors for the SCW 
components. In addition, the onset of SCW synthesis also implies that the primary wall biosynthesis needs to be terminated, which should promote re-direction of metabolic fluxes. However, our knowledge of the metabolic alterations during these events is very limited. This is surprising, given that the cell wall, and in particular the SCW, is the major carbon sink in a plant. Nevertheless, the bulk of research has focused on the immediate precursors of the cell wall polymers [68-70]. With the exception of lignin, most cell wall components are made from nucleotide sugars. Given the composition of the different polymers, it is clear that the nucleotide sugar pools must change when different cell wall polymers are made. For example, a xylan is made largely from UDP-xylose [71], whereas pectin is largely based on UDP-GalA and other nucleotide sugars depending on the type of pectin [72]. These differences raise important questions about how the carbon flux is redirected during the primary to SCW transition, and what factors control this change. Both UDP-GalA and UDP-Xyl can be generated from UDP-GlcA $[73,74]$. Thus, the flux emanating from UDP-GlcA needs to be re-routed when SCW synthesis is initiated (Fig. 1). Intriguingly, although most nucleotide interconversion reactions are reversible, the reaction from UDP-GlcA to UDP-Xyl is not. The latter is a highly exergonic process, which prevents the reaction from being reversible, and indicates a commitment of the system to irreversibly support SCW synthesis [70]. To further understand how the nucleotide sugar supply changes during the transition it will be important to explore regulatory aspects of the enzymes involved in the nucleotide sugar interconversion pathway.

Metabolic flux analyses to understand cell wall synthesis have only been undertaken in a handful of studies largely focussed at understanding starch biosynthesis in potato [75-78]. However, in the vast majority of studies neither environmental nor genetic perturbation of enzymes involved in the sucrose to starch transition had a considerable effect on cell wall biosynthesis. Nevertheless, inhibition of the expression of the nucleotide sugar biosynthetic enzyme uridine monophosphate synthase resulted in a compensatory increase in the expression of the uridine salvage pathway, an overall increase in uridinylate content and a corresponding increase in the rate of cell wall biosynthesis [76]. Additional studies, done in tomato fruit development, revealed that the carbon flux towards cell wall production decreased dramatically during later stages of fruit development [79]. It will certainly also be of interest to similarly explore carbon flux changes in the transition between PCW and SCW synthesis.

In Arabidopsis, xylem cell differentiation is concluded by lignification of the tissue [80, 81]. The mono-lignol producing framework has been extensively reviewed elsewhere [71, 82, 83], and we will only briefly summarize the pathway, and the impacts of its perturbation, here. The shikimate, the phenylpropanoid and associated pathways support lignin synthesis through a range of oxidation, methylation and hydroxylation steps [82]. Attempts to modify these pathways have provided considerable insights into the metabolic adjustments during lignin formation. Interestingly, lignin-modified plants have revealed unexpected effects on metabolic processes [84-86]. For example, silencing of hydroxycinnamoyl-CoA shikimate/quinate hydroxycinnamoyl transferase (HCT), involved in lignin precursor 
formation, in Arabidopsis caused severe growth defects and led to an accumulation of flavonoids instead of lignin [84]. These effects appear to be the result of re-routing of metabolic fluxes when enzymes in the phenylpropanoid and mono-lignol metabolism are perturbed. Therefore, it is not surprising that some changes can affect other secondary metabolites, for which precursors are intimately linked to phenylpropanoids [84, 85, 87-94]. Perhaps more interestingly, a trade-off between lignin and other cell wall polymers has been observed [95, 96], suggesting a feedback loop between the phenylpropanoid pathway and the precursor pool, or synthesis machineries, of other cell wall components. This implies either a resource distribution strategy, or a cell wall mechanical need, by the plant during SCW deposition. Nevertheless, large-scale metabolic flux studies would be necessary for a better understanding of these possible feedback processes.

While many metabolic profiling analyses have facilitated a general picture of plant growthrelated metabolic signatures [97-101], very few studies have attempted to address the metabolic changes in Arabidopsis that the primary to secondary wall transition necessitate. That said, it may be challenging to directly link metabolic changes to SCW accumulation as the tissues undergoing differentiation typically are surrounded by other cell types with different fates. This would mask metabolic changes directly linked to SCW synthesis. Nevertheless, alterations in general metabolic processes do affect SCW synthesis. For example, changes in energy metabolism alter SCW accumulation and composition. Transgenic tomato plants with altered enzyme activities associated with the tricarboxylic acid (TCA) cycle exhibited a substantial reduction in cellulose and lignin, and in xylem vessel development, in roots [102]. In this study, the authors also evaluated the metabolic flux by feeding ${ }^{[\mathrm{U}-14 \mathrm{C}]}$ Glc into the system. Under energy limited conditions, a depressed cell wall synthesis rate coupled with constant glycolysis, starch and Sucrose biosynthesis indicated that the TCA impaired plants maintained their primary metabolic pathways at the expense of other processes. Taken together, these data indicate that reduced TCA cycle activity inhibits SCW biosynthesis, linking this process to the ability of the plant to produce energy (Fig 1). While these studies provide a first insight into how changes in metabolism impact on bulk biomass production in Arabidopsis it is unclear how the metabolic fluxes proceed in a system where only primary to SCW transition is being monitored.

Apart from using Arabidopsis to address PCW to SCW transitions, valuable insights have been gained from studies on cotton fibre development. Here, the major metabolic changes seem to mainly support cellulose synthesis [103]. Decreases in glucose and fructose concentrations have been observed, which imply changes in sugar metabolism [104]. Further biochemical studies revealed that sucrose phosphate synthase may have a critical role in recycling fructose to support cellulose synthesis [105]. In addition, repression of the pectin biosynthesis pathway was also reported, supporting a low pectic content in maturing cotton fibres [103]. Furthermore, reactive oxygen species are important factors for the transition to SCW synthesis. For example, it has been shown that the cotton fibre maturation depends on a spike of $\mathrm{H}_{2} \mathrm{O}_{2}$, which might be a signal for initiating the dimerization of cellulose synthases [106]. 


\section{Tools to evaluate the primary to secondary wall transition}

In planta, certain characteristics prevent us from evaluating the primary to SCW transition easily as: the developmental programs are not synchronous in cells with the same developmental fate; SCWs are synthesized very rapidly and the transition is therefore difficult to clearly outline in a plant and, SCW-containing cells are usually located deep inside plant tissues with surrounding cells obscuring visualization and monitoring of the transitions. Nevertheless, several systems that overcome one or several of these difficulties have been developed and used.

The developmental program of wood forming tissues of trees makes them suitable to address the progression of SCW production. The cells in tree stems are arranged in a developmental order, in which different cell layers in a cross-section represent different stages of cell differentiation [107]. Therefore, using precise sampling approaches may provide snapshots of SCW progression [108], and this system has been used to perform large-scale omics analysis, such as transcriptomics and chemical profiling [109, 110]. The main shortcoming of this system is that the number of cells at each cross-section is limited, so large-scale studies and high-resolution analyses are time consuming tasks.

Trans-differentiating cell cultures offer an alternative to the tree cross-section approach. Over 30 years ago, Zinnia was used for trans-differentiation studies, i.e. induction of tracheary elements, using auxin and cytokinin as inducers [21]. Several pioneering analyses using this system dramatically improved our knowledge regarding the SCW formation, including the identification of the "master" TFs VND1-7 mentioned above [22, 25, 39, 111, 112]. These studies used transcriptomics, i.e., microarrays, to establish a time-resolved expression compendium of genes induced after trans-differentiation [22]. Here, many genes that are associated with SCW formation and regulation showed distinct induction patterns and have subsequently been shown to partake in the process. Recently, this in vitro system was further optimized by using Arabidopsis cell lines that may be trans-differentiated by adding a hormone cocktail [23]. Compared to the Zinnia system, this optimized system is more stable and efficient, with up to $40 \%$ percent of cells being synchronously trans-differentiated [23]. Studies facilitated by these cell lines have uncovered proteins that regulate a range of different aspects of SCW pattering, including many microtubule-related proteins [23, 24]. However, a limitation of the system is that in vitro stimulation cannot accurately represent the in vivo situation, particularly considering the communication between different cell types that are of great importance for lignification of tissues.

A third system has been taking advantage of the "master" TFs, mainly the VNDs, to induce trans-differentiation of cells in a plant [113]. In these systems, VND6 or VND7 were fused to the activation domain of the herpes virus VP16 protein and the glucocorticoid receptor (GR) domain [113]. Transgenic plants harbouring the constructs can then be treated with dexamethasone (DEX), a glucocorticoid derivative, to re-direct the over-expressed VND6 or VND7 to the nucleus [113]. This in vivo system is not only ideal for large-scale omics studies, but also for direct visualization of the transition [30]. In addition, these lines offer great 
potential to introgress SCW deficient mutants into them and to therefore study how the SCW synthesis process is affected when individual components are impaired. Hence, together these systems offer ways to measure metabolic re-routing during the transition of primary and SCW synthesis, and potentiate system wide analyses where metabolic and transcript changes are put in context to live cell imaging data.

Lastly, cotton fibres, which are highly elongated and thickened single cells of the seed epidermis, are excellent as a system to understand the PCW to SCW transition [114]. As the SCW thickening of cotton fibre is almost only sustained by cellulose biosynthesis it may offer a great way to better understanding the transition between primary and secondary wall cellulose synthesis. Indeed, several reports have concluded that the SCW thickening starts with a dramatic increase of cellulose synthesis during which cellulose fibrils are deposited in steep helixes relative to the fibre axis $[103,106,115,116]$. Furthermore, due to the relative ease of isolating cotton fibres and culturing cotton ovules/fibres in vitro, this system has also been employed in several systems biology studies [103, 106]. It is noteworthy that while cotton fibres are of significant economic importance, data generated from this system might not be sufficient to provide an understanding of SCW synthesis from other species as the SCW contents differ substantially. Nevertheless, these differences might become of relevance for tailoring various characteristics of cell wall properties across species barriers.

\section{Future perspectives}

Due to its potential application for biomaterial and bioenergy, SCW biology has been attracting a great deal of attention and significant progress in understanding how the synthesis is initiated and controlled has been made in the past decade. From these analyses, a complex interleaved TF-hormone regulation system has emerged. Yet, many major gaps remain: First, although it is evident that hormones play fundamental roles in triggering SCW biosynthesis, mechanistic links (e.g., direct links between hormone signalling cascades and "master switches") are largely unknown. In addition, it is also unclear whether hormone-related signaling impacts on the termination of PCW biosynthesis. Second, a detailed map of the metabolic framework that underpins SCW synthesis is lacking. A better understanding of the metabolic changes during the onset of SCW production will most likely bring new biomaterial applications, and potential approaches to increase biomass production. For example, changing enzyme activities related to cell wall synthesis have generated wood with different characteristics, suitable for diverse biomaterials [117]. Third, while transcriptional regulation has been extensively explored, translational and post-translational control of SCW formation remains tenuous. This may be especially relevant during the PCW to SCW transition as metabolic fluxes are likely to be substantially different. Lastly, while most of these relationships have been gleaned from Arabidopsis, the level of which this can be transferred to energy crop species remain unclear but is a necessary step towards achieving the goal of renewable energy and new biomaterials. 


\section{Acknowledgements}

We would like to thank Dr. Wei Zeng for useful comments on the manuscript. ZL is a recipient of MIFRS and MIRS scholarships from University of Melbourne. SP was funded by a R@MAP Professorship at University of Melbourne. We also gratefully acknowledge a grant from the European Commission's Directorate General for Research within the 7th Framework Program (FP7/2007-2013) under grant agreement 270089 (MULTIBIOPRO to $\mathrm{ARF}$ and SP).

\section{References}

[1] Smith LG (2001) Plant cell division: building walls in the right places. Nat Rev Mol Cell Biol 2:33-39

[2] De Smet I, Beeckman T (2011) Asymmetric cell division in land plants and algae: the driving force for differentiation. Nat Rev Mol Cell Biol 12:177-188

[3] Cosgrove DJ (2005) Growth of the plant cell wall. Nat Rev Mol Cell Biol 6:850-861

[4] Schuetz M, Smith R, Ellis B (2013) Xylem tissue specification, patterning, and differentiation mechanisms. J Exp Bot 64:11-31

[5] Sperry JS, Hacke UG, Pittermann J (2006) Size and function in conifer tracheids and angiosperm vessels. Am J Bot 93:1490-1500

[6] Kumar M, Campbell L, Turner S (2015) Secondary cell walls: biosynthesis and manipulation. J Exp Bot 67:515-531

[7] Zhong R, Ye ZH (2015) Secondary cell walls: biosynthesis, patterned deposition and transcriptional regulation. Plant Cell Physiol 56:195-214

[8] Doblin MS, Pettolino F, Bacic A (2010) Plant cell walls: the skeleton of the plant world. Funct Plant Biol 37:357-381

[9] Vanholme R, Morreel K, Ralph J et al (2008) Lignin engineering. Curr Opin Plant Biol 11:278-285

[10] McFarlane HE, Doring A, Persson S (2014) The cell biology of cellulose synthesis. Annu Rev Plant Biol 65:69-94

[11] Wang HZ, Dixon RA (2012) On-off switches for secondary cell wall biosynthesis. Mol Plant 5:297-303

[12] Bishopp A, Mahonen AP, Helariutta Y (2006) Signs of change: hormone receptors that regulate plant development. Development 133:1857-1869

[13] Depuydt S, Hardtke CS (2011) Hormone signalling crosstalk in plant growth regulation. Curr Biol 21:R365-R373

[14] Davies PJ (2010) The plant hormones: their nature, occurrence and functions. In:

Davies PJ ed. Plant hormones: biosynthesis, signal transduction, action! Dordrecht: Springer $1-15$

[15] Turner S, Gallois P, Brown D (2007) Tracheary element differentiation. Annu Rev Plant Biol 58:407-433

[16] Didi V, Jackson P, Hejatko J (2015) Hormonal regulation of secondary cell wall formation. J Exp Bot 66:5015-5027

[17] Demura T (2014) Tracheary element differentiation. Plant Biotechnol Rep 8:17-21

[18] Fukuda H (2004) Signals that control plant vascular cell differentiation. Nat Rev Mol Cell Biol 5:379-391

[19] Yamamoto R, Demura T, Fukuda H (1997) Brassinosteroids induce entry into the final stage of tracheary element differentiation in cultured Zinnia cells. Plant Cell Physiol 38:980-983 
[20] Fukuda H, Komamine A (1980) Direct evidence for cytodifferentiation to tracheary elements without intervening mitosis in a culture of single cells isolated from the mesophyll of Zinnia elegans. Plant Physiol 65:61-64

[21] Fukuda H, Komamine A (1980) Establishment of an experimental system for the study of tracheary element differentiation from single cells isolated from the mesophyll of Zinnia elegans. Plant Physiol 65:57-60

[22] Demura T, Tashiro G, Horiguchi G et al (2002) Visualization by comprehensive microarray analysis of gene expression programs during transdifferentiation of mesophyll cells into xylem cells. Proc Natl Acad Sci U S A 99:15794-15799

[23] Pesquet E, Korolev AV, Calder G et al (2010) The microtubule-associated protein AtMAP70-5 regulates secondary wall patterning in Arabidopsis wood cells. Curr Biol 20:744-749

[24] Derbyshire P, Menard D, Green P et al (2015) Proteomic analysis of microtubule interacting proteins over the course of xylem tracheary element formation in Arabidopsis. Plant Cell 27:2709-2726

[25] Kubo M, Udagawa M, Nishikubo N et al (2005) Transcription switches for protoxylem and metaxylem vessel formation. Genes \& Development 19:1855-1860

[26] Jensen MK, Kjaersgaard T, Nielsen MM et al (2010) The Arabidopsis thaliana NAC transcription factor family: structure-function relationships and determinants of ANAC019 stress signalling. Biochem J 426:183-196

[27] Soyano T, Thitamadee S, Machida Y et al (2008) ASYMMETRIC LEAVES2LIKE19/LATERAL ORGAN BOUNDARIES DOMAIN30 and ASL20/LBD18 regulate tracheary element differentiation in Arabidopsis. Plant Cell 20:3359-3373

[28] Mutwil M, Debolt S, Persson S (2008) Cellulose synthesis: a complex complex. Curr Opin Plant Biol 11:252-257

[29] Endler A, Persson S (2011) Cellulose synthases and synthesis in Arabidopsis. Mol Plant 4:199-211

[30] Watanabe Y, Meents MJ, McDonnell LM et al (2015) Visualization of cellulose synthases in Arabidopsis secondary cell walls. Science 350:198-203

[31] Paredez AR, Somerville CR, Ehrhardt DW (2006) Visualization of cellulose synthase demonstrates functional association with microtubules. Science 312:1491-1495

[32] Sampathkumar A, Gutierrez R, McFarlane HE et al (2013) Patterning and lifetime of plasma membrane-localized cellulose synthase is dependent on actin organization in Arabidopsis interphase cells. Plant Physiol 162:675-688

[33] Li SD, Lei L, Somerville CR et al (2012) Cellulose synthase interactive protein 1 (CSI1) links microtubules and cellulose synthase complexes. Proc Natl Acad Sci USA 109:185-190

[34] Mei Y, Gao HB, Yuan M et al (2012) The Arabidopsis ARCP protein, CSI1, which Is required for microtubule stability, is necessary for root and anther development. Plant Cell 24:1066-1080

[35] Bringmann M, Li EY, Sampathkumar A et al (2012) POM-POM2/CELLULOSE SYNTHASE INTERACTING1 is essential for the functional association of cellulose synthase and microtubules in Arabidopsis. Plant Cell 24:163-177

[36] Sedbrook JC, Kaloriti D (2008) Microtubules, MAPs and plant directional cell expansion. Trends Plant Sci 13:303-310

[37] Oda Y, Fukuda H (2012) Secondary cell wall patterning during xylem differentiation. Curr Opin Plant Biol 15:38-44

[38] Crowell EF, Bischoff V, Desprez T et al (2009) Pausing of golgi bodies on microtubules regulates secretion of cellulose synthase complexes in Arabidopsis. Plant Cell 21:1141-1154 
[39] Oda Y, Mimura T, Hasezawa S (2005) Regulation of secondary cell wall development by cortical microtubules during tracheary element differentiation in Arabidopsis cell suspensions. Plant Physiol 137:1027-1036

[40] Bouquin T, Mattsson O, Naested H et al (2003) The Arabidopsis luel mutant defines a katanin p60 ortholog involved in hormonal control of microtubule orientation during cell growth. J Cell Sci 116:791-801

[41] Catterou M, Dubois F, Schaller H et al (2001) Brassinosteroids, microtubules and cell elongation in Arabidopsis thaliana. I. Molecular, cellular and physiological characterization of the Arabidopsis bull mutant, defective in the delta(7)-sterol-C5-desaturation step leading to brassinosteroid biosynthesis. Planta 212:659-672

[42] Xu T, Wen M, Nagawa S et al (2010) Cell surface- and Rho GTPase-based auxin signaling controls cellular interdigitation in Arabidopsis. Cell 143:99-110

[43] Blancaflor EB, Hasenstein KH (1993) Organization of cortical microtubules in graviresponding maize roots. Planta 191:231-237

[44] Blancaflor EB, Hasenstein KH (1995) Time-course and auxin sensitivity of cortical microtubule reorientation in maize roots. Protoplasma 185:72-82

[45] Takesue K, Shibaoka H (1998) The cyclic reorientation of cortical microtubules in epidermal cells of azuki bean epicotyls: the role of actin filaments in the progression of the cycle. Planta 205:539-546

[46] Takesue K, Shibaoka H (1999) Auxin-induced longitudinal-to-transverse reorientation of cortical microtubules in nonelongating epidermal cells of azuki bean epicotyls.

Protoplasma 206:27-30

[47] Baluska F, Barlow PW, Volkmann D (1996) Complete disintegration of the microtubular cytoskeleton precedes its auxin-mediated reconstruction in postmitotic maize root cells. Plant Cell Physiol 37:1013-1021

[48] Chen X, Grandont L, Li H et al (2014) Inhibition of cell expansion by rapid ABP1mediated auxin effect on microtubules. Nature 516:90-U206

[49] Gao YB, Zhang Y, Zhang D et al (2015) Auxin binding protein 1 (ABP1) is not required for either auxin signaling or Arabidopsis development. Proc Natl Acad Sci USA $112: 2275-2280$

[50] Dai XH, Zhang Y, Zhang D et al (2015) Embryonic lethality of Arabidopsis abp1-1 is caused by deletion of the adjacent BSM gene. Nat Plants 1: 15183

[51] Schopfer P, Palme K (2016) Inhibition of cell expansion by rapid ABP1-mediated auxin effect on microtubules? A critical comment. Plant physiol 170:23-25

[52] Endler A, Kesten C, Schneider R et al (2015) A mechanism for sustained cellulose synthesis during salt stress. Cell 162:1353-1364

[53] Taylor-Teeples M, Lin L, de Lucas M et al (2015) An Arabidopsis gene regulatory network for secondary cell wall synthesis. Nature 517:571-575

[54] Zhong RQ, Ye ZH (2014) Complexity of the transcriptional network controlling secondary wall biosynthesis. Plant Sci 229:193-207

[55] Nakano Y, Yamaguchiz M, Endo H et al (2015) NAC-MYB-based transcriptional regulation of secondary cell wall biosynthesis in land plants. Front Plant Sci 6:

[56] Zhong R, Ye Z-H (2007) Regulation of cell wall biosynthesis. Curr Opin Plant Biol 10:564-572

[57] Demura T, Fukuda H (2007) Transcriptional regulation in wood formation. Trends in Plant Science 12:64-70

[58] Hussey SG, Mizrachi E, Creux NM et al (2013) Navigating the transcriptional roadmap regulating plant secondary cell wall deposition. Front Plant Sci 4:325 
[59] Yamaguchi M, Kubo M, Fukuda $\mathrm{H}$ et al (2008) VASCULAR-RELATED NACDOMAIN7 is involved in the differentiation of all types of xylem vessels in Arabidopsis roots and shoots. Plant Journal 55:652-664

[60] Yamaguchi M, Mitsuda N, Ohtani M et al (2011) VASCULAR-RELATED NACDOMAIN 7 directly regulates the expression of a broad range of genes for xylem vessel formation. Plant Journal 66:579-590

[61] Zhou J, Zhong R, Ye Z-H (2014) Arabidopsis NAC domain proteins, VND1 to VND5, are transcriptional regulators of secondary wall biosynthesis in vessels. Plos One 9: e105726

[62] Mitsuda N, Seki M, Shinozaki K et al (2005) The NAC transcription factors NST1 and NST2 of Arabidopsis regulate secondary wall thickenings and are required for anther dehiscence. Plant Cell 17:2993-3006

[63] Zhong R, Demura T, Ye Z-H (2006) SND1, a NAC domain transcription factor, is a key regulator of secondary wall synthesis in fibers of Arabidopsis. Plant Cell 18:3158-3170 [64] Mitsuda N, Iwase A, Yamamoto H et al (2007) NAC transcription factors, NST1 and NST3, are key regulators of the formation of secondary walls in woody tissues of Arabidopsis. Plant Cell 19:270-280

[65] Wang H, Zhao Q, Chen F et al (2011) NAC domain function and transcriptional control of a secondary cell wall master switch. Plant J 68:1104-1114

[66] Yamaguchi M, Ohtani M, Mitsuda N et al (2010) VND-INTERACTING2, a NAC domain transcription factor, negatively regulates xylem vessel formation in Arabidopsis. Plant Cell 22:1249-1263

[67] Wang H, Avci U, Nakashima J et al (2010) Mutation of WRKY transcription factors initiates pith secondary wall formation and increases stem biomass in dicotyledonous plants. Proc Natl Acad Sci USA 107:22338-22343

[68] Amor Y, Haigler CH, Johnson S et al (1995) A membrane-associated form of sucrose synthase and its potential role in synthesis of cellulose and callose in plants. Proc Natl Acad Sci USA 92:9353-9357

[69] Ruan YL, Llewellyn DJ, Furbank RT (2003) Suppression of sucrose synthase gene expression represses cotton fiber cell initiation, elongation, and seed development. Plant Cell 15:952-964

[70] Reiter WD (2008) Biochemical genetics of nucleotide sugar interconversion reactions. Curr Opin Plant Biol 11:236-243

[71] Hao Z, Mohnen D (2014) A review of xylan and lignin biosynthesis: foundation for studying Arabidopsis irregular xylem mutants with pleiotropic phenotypes. Crit Rev Biochem Mol Biol 49:212-241

[72] Atmodjo MA, Hao ZY, Mohnen D (2013) Evolving views of pectin biosynthesis. Annu Rev Plant Biol 64:747-779

[73] Pattathil S, Harper AD, Bar-Peled M (2005) Biosynthesis of UDP-xylose: characterization of membrane-bound At Uxs2. Planta 221:538-548

[74] Litterer LA, Schnurr JA, Plaisance KL et al (2006) Characterization and expression of Arabidopsis UDP-sugar pyrophosphorylase. Plant Physiol Biochem 44:171-180

[75] Geigenberger P, Fernie AR, Gibon Y et al (2000) Metabolic activity decreases as an adaptive response to low internal oxygen in growing potato tubers. Biol Chem 381:723-740

[76] Geigenberger P, Regierer B, Nunes-Nesi A et al (2005) Inhibition of de novo pyrimidine synthesis in growing potato tubers leads to a compensatory stimulation of the pyrimidine salvage pathway and a subsequent increase in biosynthetic performance. Plant Cell 17:2077-2088

[77] Fernie AR, Roessner U, Trethewey RN et al (2001) The contribution of plastidial phosphoglucomutase to the control of starch synthesis within the potato tuber. Planta 213:418-426 
[78] Oliver SN, Lunn JE, Urbanczyk-Wochniak E et al (2008) Decreased expression of cytosolic pyruvate kinase in potato tubers leads to a decline in pyruvate resulting in an in vivo repression of the alternative oxidase. Plant Physiol 148:1640-1654

[79] Carrari F, Baxter C, Usadel B et al (2006) Integrated analysis of metabolite and transcript levels reveals the metabolic shifts that underlie tomato fruit development and highlight regulatory aspects of metabolic network behavior. Plant Physiol 142:1380-1396

[80] Voxeur A, Wang Y, Sibout R (2015) Lignification: different mechanisms for a versatile polymer. Curr Opin Plant Biol 23:83-90

[81] Barros J, Serk H, Granlund I et al (2015) The cell biology of lignification in higher plants. Ann Bot 115:1053-1074

[82] Boerjan W, Ralph J, Baucher M (2003) Lignin biosynthesis. Annu Rev Plant Biol 54:519-546

[83] Zhao Q, Dixon RA (2011) Transcriptional networks for lignin biosynthesis: more complex than we thought? Trends Plant Sci 16:227-233

[84] Besseau S, Hoffmann L, Geoffroy P et al (2007) Flavonoid accumulation in Arabidopsis repressed in lignin synthesis affects auxin transport and plant growth. Plant Cell 19:148-162

[85] Coleman HD, Park JY, Nair R et al (2008) RNAi-mediated suppression of pcoumaroyl-CoA 3 '-hydroxylase in hybrid poplar impacts lignin deposition and soluble secondary metabolism. Proc Natl Acad Sci USA 105:4501-4506

[86] Vanholme R, Storme V, Vanholme B et al (2012) A systems biology view of responses to lignin biosynthesis perturbations in Arabidopsis. Plant Cell 24:3506-3529

[87] Gallego-Giraldo L, Jikumaru Y, Kamiya Y et al (2011) Selective lignin downregulation leads to constitutive defense response expression in alfalfa (Medicago sativa L.). New Phytol 190:627-639

[88] Meyermans H, Morreel K, Lapierre C et al (2000) Modifications in lignin and accumulation of phenolic glucosides in poplar xylem upon down-regulation of caffeoylcoenzyme A O-methyltransferase, an enzyme involved in lignin biosynthesis. J Biol Chem 275:36899-36909

[89] Chen F, Duran AL, Blount JW et al (2003) Profiling phenolic metabolites in transgenic alfalfa modified in lignin biosynthesis. Phytochemistry 64:1013-1021

[90] Lanot A, Hodge D, Lim EK et al (2008) Redirection of flux through the phenylpropanoid pathway by increased glucosylation of soluble intermediates. Planta 228:609-616

[91] Quentin M, Allasia V, Pegard A et al (2009) Imbalanced lignin biosynthesis promotes the sexual reproduction of homothallic oomycete pathogens. Plos Pathog 5: e1000264 [92] Goujon T, Sibout R, Pollet B et al (2003) A new Arabidopsis thaliana mutant deficient in the expression of $O$-methyltransferase impacts lignins and sinapoyl esters. Plant Mol Biol 51:973-989

[93] Fornale S, Shi X, Chai C et al (2010) ZmMYB31 directly represses maize lignin genes and redirects the phenylpropanoid metabolic flux. Plant J 64:633-644

[94] Sonbol F-M, Fornale S, Capellades M et al (2009) The maize ZmMYB42 represses the phenylpropanoid pathway and affects the cell wall structure, composition and degradability in Arabidopsis thaliana. Plant Mol Biol 70:283-296

[95] Van Acker R, Vanholme R, Storme V et al (2013) Lignin biosynthesis perturbations affect secondary cell wall composition and saccharification yield in Arabidopsis thaliana. Biotechnol Biofuels 6:46

[96] Van Acker R, Leple JC, Aerts D et al (2014) Improved saccharification and ethanol yield from field-grown transgenic poplar deficient in cinnamoyl-CoA reductase. Proc Natl Acad Sci USA 111:845-850 
[97] Meyer RC, Steinfath M, Lisec J et al (2007) The metabolic signature related to high plant growth rate in Arabidopsis thaliana. Proc Natl Acad Sci USA 104:4759-4764

[98] Sulpice R, Pyl ET, Ishihara H et al (2009) Starch as a major integrator in the regulation of plant growth. Proc Natl Acad Sci USA 106:10348-10353

[99] Smeekens S, Ma JK, Hanson J et al (2010) Sugar signals and molecular networks controlling plant growth. Curr Opin Plant Biol 13:274-279

[100] Schluepmann H, Berke L, Sanchez-Perez GF (2012) Metabolism control over growth: a case for trehalose-6-phosphate in plants. J Exp Bot 63:3379-3390

[101] O'Hara LE, Paul MJ, Wingler A (2013) How do sugars regulate plant growth and development? new insight into the role of trehalose-6-phosphate. Mol Plant 6:261-274 [102] van der Merwe MJ, Osorio S, Araujo WL et al (2010) Tricarboxylic acid cycle activity regulates tomato root growth via effects on secondary cell wall production. Plant Physiol 153:611-621

[103] Gou JY, Wang LJ, Chen SP et al (2007) Gene expression and metabolite profiles of cotton fiber during cell elongation and secondary cell wall synthesis. Cell Res 17:422-434 [104] Martin LK, Haigler CH (2004) Cool temperature hinders flux from glucose to sucrose during cellulose synthesis in secondary wall stage cotton fibers. Cellulose 11:339-349

[105] Babb VM, Haigler CH (2001) Sucrose phosphate synthase activity rises in correlation with high-rate cellulose synthesis in three heterotrophic systems. Plant Physiol 127:12341242

[106] Tuttle JR, Nah G, Duke MV et al (2015) Metabolomic and transcriptomic insights into how cotton fiber transitions to secondary wall synthesis, represses lignification, and prolongs elongation. BMC Genomics 16:

[107] Mellerowicz EJ, Baucher M, Sundberg B et al (2001) Unravelling cell wall formation in the woody dicot stem. Plant Mol Biol 47:239-274

[108] Uggla C, Moritz T, Sandberg G et al (1996) Auxin as a positional signal in pattern formation in plants. Proc Natl Acad Sci USA 93:9282-9286

[109] Schrader J, Nilsson J, Mellerowicz E et al (2004) A high-resolution transcript profile across the wood-forming meristem of poplar identifies potential regulators of cambial stem cell identity. Plant Cell 16:2278-2292

[110] Gorzsas A, Stenlund H, Persson P et al (2011) Cell-specific chemotyping and multivariate imaging by combined FT-IR microspectroscopy and orthogonal projections to latent structures (OPLS) analysis reveals the chemical landscape of secondary xylem. Plant $\mathrm{J}$ 66:903-914

[111] Milioni D, Sado PE, Stacey NJ et al (2002) Early gene expression associated with the commitment and differentiation of a plant tracheary element is revealed by cDNA-amplified fragment length polymorphism analysis. Plant Cell 14:2813-2824

[112] Pesquet E, Ranocha P, Legay S et al (2005) Novel markers of xylogenesis in zinnia are differentially regulated by auxin and cytokinin. Plant Physiol 139:1821-1839

[113] Yamaguchi M, Goue N, Igarashi H et al (2010) VASCULAR-RELATED NACDOMAIN6 and VASCULAR-RELATED NAC-DOMAIN7 effectively induce transdifferentiation into xylem vessel elements under control of an induction system. Plant Physiol 153:906-914

[114] Haigler CH, Betancur L, Stiff MR et al (2012) Cotton fiber: a powerful single-cell model for cell wall and cellulose research. Front Plant Sci 3:104

[115] Abidi N, Hequet E, Cabrales L (2010) Changes in sugar composition and cellulose content during the secondary cell wall biogenesis in cotton fibers. Cellulose 17:153-160 [116] Singh B, Avci U, Inwood SEE et al (2009) A specialized outer layer of the primary cell wall joins elongating cotton fibers into tissue-like bundles. Plant Physiol 150:684-699 
[117] Goswami L, Eder M, Gierlinger N et al (2008) Inducing large deformation in wood cell walls by enzymatic modification. J Mater Sci 43:1286-1291 
Fig. 1 Hormonal and transcriptional control, and metabolic adjustment, associated with the transition from PCW to SCW synthesis. A complex transcriptional network is employed by plants to regulate the SCW biosynthetic program. In Arabidopsis, several tissuespecific master TFs, namely, VND1-7, NST1/2 and SND1, have the capacity to trigger transdifferentiation of cells. Upstream regulators, and multiple levels of downstream TFs, comprise an intricate feed-forward /feedback loop system that control the expression of SCW biosynthetic genes. Several hormones control the expression of the master TFs via unknown signalling pathways or other TF tiers (e.g., ASL19/20). During the transition, hormones might also impact on the microtubules re-organization. To rapidly synthesize SCWs, the flux of carbon needs to be redirected through the nucleotide sugar pool as different cell wall polymers need different precursors. In addition, altered primary metabolism (e.g., TCA cycle) is required to provide energy for the $\mathrm{PCW}$ to SCW transition. Please note that the relative order and alignment between the different processes is largely unknown. ABA: abscisic acid; AUX: auxin; CK: cytokinin; BR: brassinosteroid; GA: gibberellic acid.

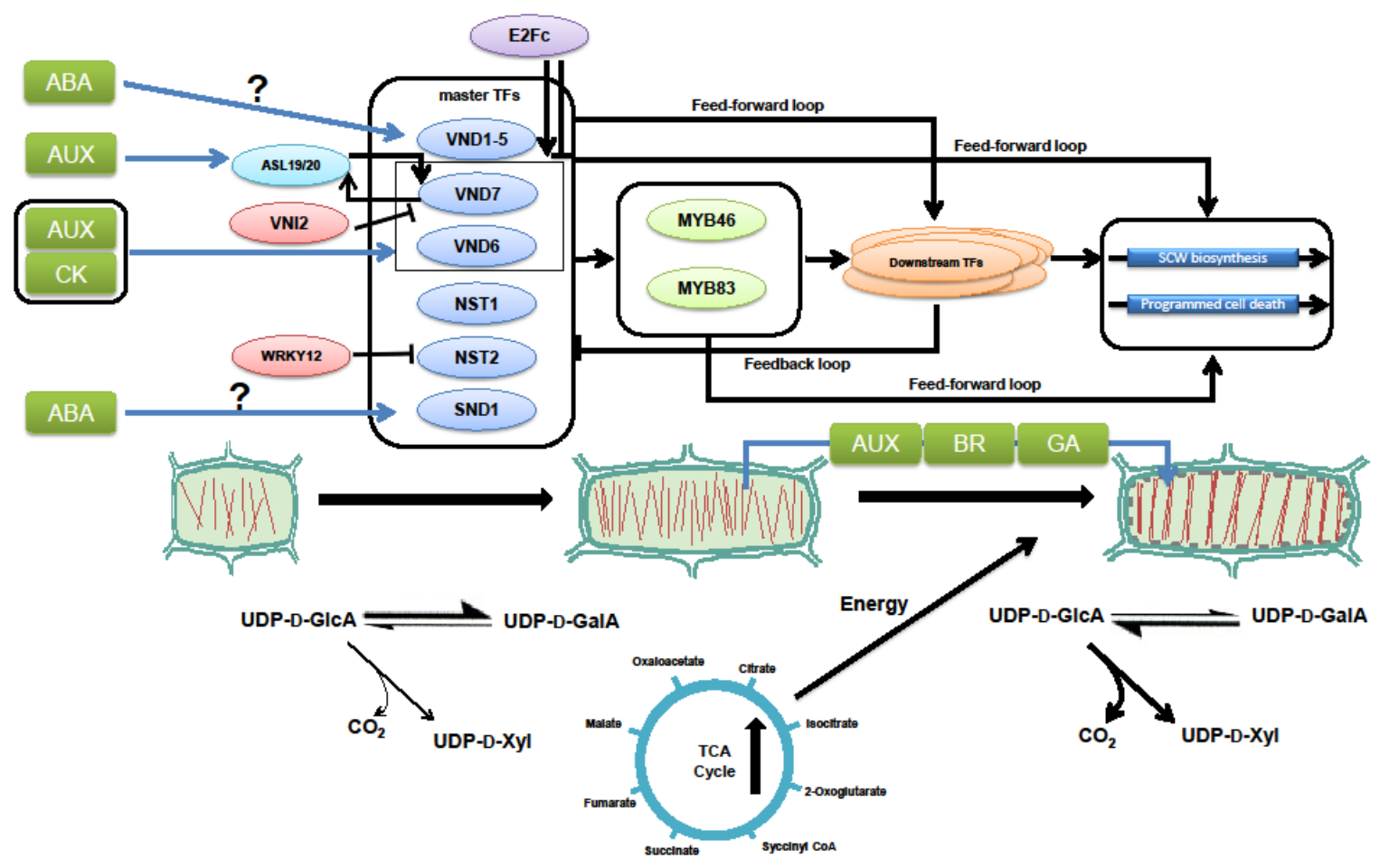




\section{University Library}

\section{- M M I N E R VA A gateway to Melbourne's research publications}

Minerva Access is the Institutional Repository of The University of Melbourne

Author/s:

Li, Z;Fernie, AR;Persson, S

Title:

Transition of primary to secondary cell wall synthesis

Date:

2016-06-01

Citation:

Li, Z., Fernie, A. R. \& Persson, S. (2016). Transition of primary to secondary cell wall synthesis. SCIENCE BULLETIN, 61 (11), pp.838-846. https://doi.org/10.1007/ s11434-016-1061-7.

Persistent Link:

http://hdl.handle.net/11343/282576 